\title{
MHD model of magnetosheath flow: comparison with AMPTE/IRM observations on 24 October, 1985
}

\author{
C. J. Farrugia ${ }^{1}$, H. K. Biernat ${ }^{2}$, N. V. Erkaev ${ }^{3}$, L. M. Kistler ${ }^{1}$, G. Le ${ }^{4}$, C. T. Russell ${ }^{4}$ \\ ${ }^{1}$ Institute for the Study of Earth, Oceans and Space, University New Hampshire, Durham, NH 03824 \\ ${ }^{2}$ Space Research Institute, Austrian Academy of Sciences, A-8042, Graz, Austria \\ ${ }^{3}$ Institute of Computational Modelling of the Russian Academy of Sciences, Siberian Branch, Krasnoyarsk, 660036, Russia \\ ${ }^{4}$ Institute of Geophysics and Planetary Physics, University of California, Los Angeles, CA 90032
}

Received 30 April 1997 / Revised 5 December 1997 / Accepted 9 January 1998

\begin{abstract}
We compare numerical results obtained from a steady-state MHD model of solar wind flow past the terrestrial magnetosphere with documented observations made by the AMPTE/IRM spacecraft on 24 October, 1985, during an inbound crossing of the magnetosheath. Observations indicate that steady conditions prevailed during this about 4 hour-long crossing. The magnetic shear at spacecraft entry into the magnetosphere was $15^{\circ}$. A steady density decrease and a concomitant magnetic field pile-up were observed during the $40 \mathrm{~min}$ interval just preceding the magnetopause crossing. In this plasma depletion layer (1) the plasma beta dropped to values below unity; (2) the flow speed tangential to the magnetopause was enhanced; and (3) the local magnetic field and velocity vectors became increasingly more orthogonal to each other as the magnetopause was approached (Phan et al., 1994). We model parameter variations along a spacecraft orbit approximating that of AMPTE/IRM, which was at slightly southern GSE latitudes and about $1.5 \mathrm{~h}$ postnoon Local Time. We model the magnetopause as a tangential discontinuity, as suggested by the observations, and take as input solar wind parameters those measured by AMPTE/IRM just prior to its bow shock crossing. We find that computed field and plasma profiles across the magnetosheath and plasma depletion layer match all observations closely. Theoretical predictions on stagnation line flow near this low-shear magnetopause are confirmed by the experimental findings. Our theory does not give, and the data on this pass do not show, any localized density enhancements in the inner magnetosheath region just outside the plasma depletion layer.
\end{abstract}

Key words. Steady-state magnetosheath $\cdot$ Plasma depletion layer $\cdot$ Stagnation line flow

\section{Introduction}

In the region adjacent to the sunward side of the dayside magnetopause, spacecraft often encounter a layer where the magnetic field and plasma parameters show a behaviour markedly different from that in the rest of the magnetosheath: as the magnetopause is approached, the magnetic field strength, $B$, is enhanced and simultaneously the plasma density, $N$, is depressed with respect to values in the rest of the magnetosheath. This is the plasma depletion layer (PDL). Among other properties of the PDL are (1) plasma flows whose component tangential to the magnetopause is enhanced and which rotate to align themselves more nearly perpendicular to the local magnetic field at the magnetopause; and (2) the occurrence of electromagnetic ion cyclotron wave emissions. The former is evidence of stagnation line flow features (see later). The latter serves as a further characterization of the layer, in addition to the $B$ and $N$ behaviour. Observational evidence for the PDL was first reported by Paschmann et al. (1978) and Crooker et al. (1979). It has subsequently often been observed, and its properties studied, by AMPTE experimenters (Anderson et al., 1991; Paschmann et al., 1993; Phan et al., 1994; Phan and Paschmann, 1995).

Theoretical studies which include the influence of the interplanetary magnetic field (IMF) on magnetosheath flow have long predicted the existence of the PDL (Midgley and Davies, 1963; Lees, 1964; Zwan and Wolf, 1976; Erkaev, 1988). Physically, the PDL arises from the stretching of magnetosheath field lines as the magnetosheath plasma is diverted past the magnetospheric obstacle, particularly when the IMF has a component perpendicular to the solar wind bulk flow. (If the IMF direction is along the bulk flow, no PDL forms and the flow can be described by gas dynamics, Farrugia et al., 1997a.) This process results in a compression and draping of the magnetic field about the magnetospheric obstacle and an acceleration of plasma perpendicular to 
the field. Reconnection between the magnetosheath and terrestrial magnetic fields weakens the depletion effect since, by enabling plasma to escape in high-speed jets along the magnetopause, it inhibits a large magnetic field pile-up in front of the magnetopause. Gas dynamics, which does not include the magnetic forces acting on the flow, cannot make any contribution to the PDL as commonly understood.

A systematic study of low-latitude magnetosheath crossings made by the AMPTE/IRM spacecraft shows that the PDL is present when the local magnetic field shear across the magnetopause, angle $\psi$, is low $\left(\leq 30^{\circ}\right)$, and is absent when $\psi$ is high (Paschmann et al., 1993; Phan et al., 1994; Phan and Paschmann, 1995). The plasma and magnetic field behaviour in the near-magnetopause magnetosheath is found to be strongly dependent on $\psi$. This, in turn, is evidence that transfer processes at the low-shear magnetopause are very different from those at the high-shear magnetopause (Paschmann et al., 1993; Phan et al., 1994; Phan and Paschmann, 1995).

In addition to the local magnetic shear across the magnetopause, the PDL depends sensitively also on the Alfvén Mach number upstream of the bow shock, $M_{A \infty}$ (Erkaev, 1988; Farrugia et al., 1995, 1997a,b), since $M_{A \infty}$ fixes the boundary condition at the bow shock needed to integrate the magnetosheath flow equations. The limiting case $M_{A \infty} \longrightarrow \infty$ corresponds to gas dynamics, where magnetic forces are neglected. As $M_{A \infty}$ decreases, the IMF exerts an increasing influence on the flow. Very low $M_{A \infty}$ lead to wide PDLs. For reasonable values of the reconnection rate, a PDL has been hypothesized even when $\psi$ is large (Farrugia et al., 1995, 1997b), and an example confirming this has also been reported (Farrugia et al., 1997a).

Peculiar to our MHD predictions of magnetosheath flow near a tangential discontinuity magnetopause is stagnation line flow. Figure 1 shows computed flow streamlines at the sunward side of the magnetopause for an IMF vector pointing along the arrow shown at the top of the figure (after Farrugia et al., 1995). (If the IMF vector rotates in the $(y, z)$ plane, the whole pattern rotates with it, remaining symmetrical about the field direction.) In gas dynamics, the flow streamlines would diverge radially away from the stagnation point at the centre of the figure. Inclusion of the magnetic field, however, results in a force being exerted on the flow perpendicular to the field causing the plasma to accelerate in the direction orthogonal to the field and tangential to the magnetopause. The streamlines then curve away from the direction of the reference magnetic field at the sunward side of the magnetopause. This stagnation line flow pattern, which is appropriate for low-shear magnetopauses, was first proposed by Sonnerup (1974) and Pudovkin and Semenov (1977) as a means of avoiding singularities in the field and plasma behaviour at the magnetopause.

It is the purpose of this work to compare the results of a numerical code which integrates the steady-state ideal MHD equations under simplifying assumptions with observations made on 24 October, 1985 by AMPTE/IRM as the spacecraft traversed the dayside mag-
Flow streamlines at the magnetopause
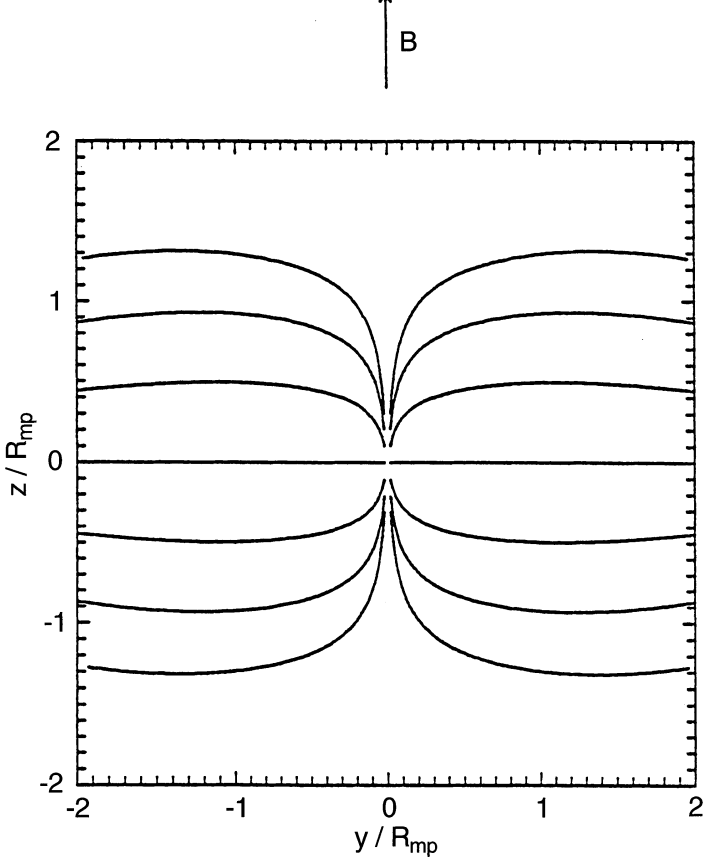

Fig. 1. Magnetosheath stagnation line flow computed for $\psi=0$ and $M_{A \infty}=3$

netosheath at low latitudes and crossed the low-shear magnetopause. This pass, one of 13 low-shear cases studied by Phan et al. (1994), has been chosen for two reasons. The first reason is that magnetosheath conditions were inferred to be stationary (Phan et al., 1994). While our code has been applied to a non-steady solar wind input (Farrugia et al., 1997a), stationary conditions are best suited to illustrate PDL features, which is our main purpose here. The second reason is that the spacecraft was in the PDL for about $40 \mathrm{~min}$ of an approx 4-h magnetosheath crossing, i.e., the PDL constituted about $1 / 6$ of the low-latitude magnetosheath thickness under the conditions prevailing during the pass. This is about four times as long as the average duration of PDL traversals in the AMPTE/IRM survey (Phan et al., 1994; Phan and Paschmann, 1995). We shall find that theoretical predictions agree with trends in the field and plasma variations both across the magnetosheath proper and in the PDL, and reproduce the actual measurements quite closely. We shall also show that the predicted stagnation line flow is confirmed by the observations.

\section{MHD numerical code and simplifications}

The procedure for solving the MHD equations describing magnetosheath flow has been described in detail by Erkaev (1988), Biernat et al. (1995), and Farrugia et al. (1995), and only a brief outline is given here. The starting point is the usual set of ideal MHD equations with a polytropic equation of state of index $\gamma=5 / 3$. For computational purposes, these equations are re-cast into 
"magnetic string" equations (Erkaev, 1988; Biernat et al., 1995; Farrugia et al., 1995), the name deriving from a formal analogy between the resulting equations and those describing the vibrations of a string. Boundary conditions at the bow shock are provided by the usual Rankine-Hugoniot equations expressing conservation laws at the shock (Spreiter et al., 1966). In line with the observations (Phan et al., 1994), we model the magnetopause during this pass as a tangential discontinuity, i.e., with vanishing normal flow and field components $\left(V_{n}=B_{n}=0\right)$.

The magnetosphere is modelled as a dipole confined within a paraboloid of revolution. The total pressure $\Pi$ (i.e., the sum of the thermal and magnetic pressures) is assumed to vary along the magnetopause surface in accordance with the Newtonian formula $\Pi=$ $\left(\Pi^{(o)}-\Pi_{\infty}\right) \cos ^{2} \theta+\Pi_{\infty}$, where indices "o" and " $\infty$ " refer to quantities at the stagnation point and in the upstream medium, respectively, and $\theta$ denotes the angle between the stagnation streamline and the normal to the magnetopause. This relation is the same as the third and preferred formula for the pressure variation along the surface of a planetary obstacle discussed by Petrinec and Russell (1997).
In the numerical integration of the MHD equations, we make a simplifying assumption concerning the variation of quantity $\Pi$ along any given normal to the model magnetosphere. The numerical integration scheme requires this variation to be a prescribed function of distance from the magnetopause along the normal, $s$. In the present application, we take $\Pi$ to vary quadratically with $s: \quad \Pi=\Pi_{m}\left(1-s^{2} / \delta^{2}\right)+\Pi_{s} s^{2} / \delta^{2}$, where $\delta$ is the thickness of the magnetosheath, and $\Pi_{s}$, $\Pi_{m}$ are the total pressures just downstream of the bow shock and on the obstacle, respectively. This relation is a model assumption, but we shall show it to be in good agreement with the data on this pass. The coefficients in the quadratic relation are obtained from conditions $\Pi$ must satisfy at the bow shock and at the magnetopause.

\section{Magnetosheath crossing on 24, October, 1985: observations}

The pass we shall examine is the inbound AMPTE/IRM crossing of the magnetosheath on October 24, 1985. Phan et al. (1994) concluded that the conditions were quasi-stationary (see later). We check this by showing in
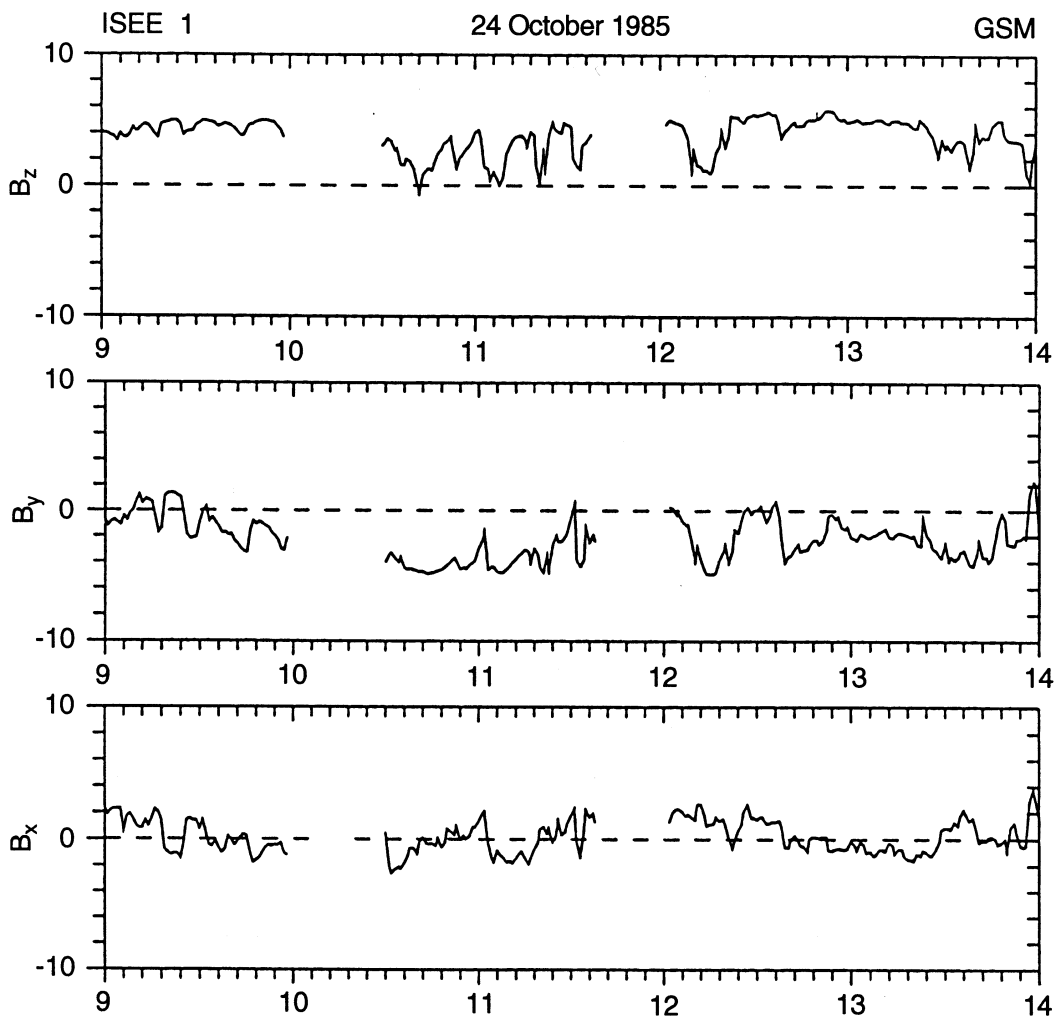

$\infty$

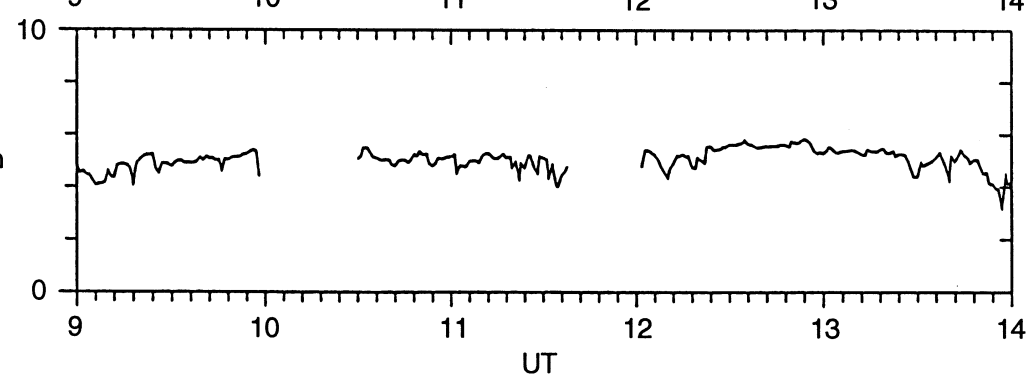

Fig. 2. IMF data from ISEE 1, situated near the Sun-Earth line a few $R_{e}$ upstream of AMPTE/IRM 
Fig. 2 IMF measurements made by ISEE 1, which was upstream of the bow shock close to the Earth-Sun line at $(19.2,0.8,-1.2) \quad R_{e}$ and $(20.7,2.0,0) R_{e}$ (GSE coordinates) at 9 and 13 UT, respectively. The components of the magnetic field (GSM coordinates) and the magnetic field strength are plotted at 1 min temporal resolution. (Plasma data from the Fast Plasma Experiment on ISEE 1 are not shown as this instrument is not designed for solar wind measurements.) By comparing the $B_{y}$ and $B_{z}$ components on ISEE 1 and AMPTE/IRM (see Fig. 3) between 12 and 13 UT, we arrive at a signal propagation delay of $\sim 6 \mathrm{~min}$. Figure 2 shows a fairly constant field of $\sim 5 \mathrm{nT}$, with a small $B_{x}$ component, and generally large positive $B_{z}$ and negative $B_{y}$ components. There are no interplanetary shocks present, but some rotations in the field in the $(y z)$ plane are evident. The $B_{y}$ and $B_{z}$ field components are both orthogonal to the bulk flow velocity and in our MHD model they give equal contributions to PDL structure, e.g., they are both compressed by the same amount. We shall show later when we discuss Fig. 3 that this is borne out by AMPTE/IRM data. The ISEE 1 data thus support the stationarity inference made from AMPTE/IRM data, and shall henceforth be adopted.

Figure 3 shows AMPTE/IRM magnetic field observations on this pass, and displays from top to bottom the $B_{z}, B_{y}$ and $B_{x}$ components of the magnetic field (in $\mathrm{nT}$; GSM coordinates) and the total field strength (nT) for the time interval from 9 to 14 UT. All components are plotted on the same scale. The bow shock and magnetopause are crossed at $\sim 9: 14$ UT and 13:03 UT, respectively. There is a brief excursion into the solar wind from 09:36 to 09:40 UT, indicative of some variability in the interplanetary medium at this time. The crossing of the low-shear magnetopause $\left(15^{\circ}\right.$ locally, Phan et al., 1994) is inferred from rapid increases in the electron (not shown) and proton temperatures (see Fig. 6) which occur there and which provide a reliable identification of low-shear magnetopause crossings (Paschmann et al., 1993). The spacecraft follows a trajectory which is on average along the 13:30 LT (GSE) line and $\sim 5^{\circ}$ south of the ecliptic plane.

A prominent feature of the data in Fig. 3 is the increase in average magnetic field strength during the second part of the crossing. The magnetic field strength at the magnetopause is about 3.5 times its value just downstream of the bow shock. The strongest gradients in the magnetic field strength profile occur in the last few tens of minutes prior to the magnetopause crossing (from 12:24 UT to 13:03 UT), with the largest increases being in the $B_{z}$ and $B_{x}$ components. A comparison with IMF data in Fig. 2 shows that the magnetosheath

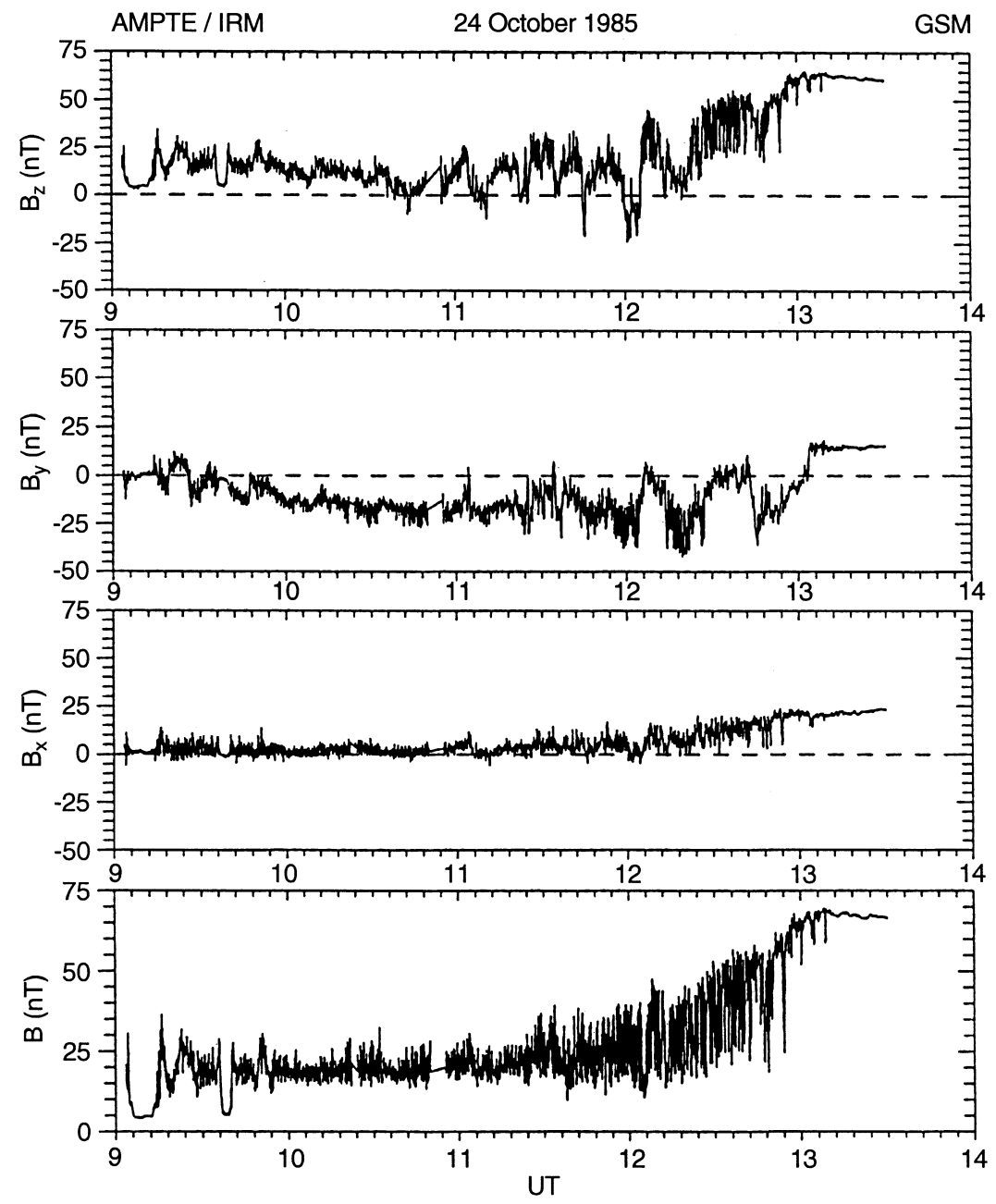

Fig. 3. The inbound magnetosheath crossing by AMPTE/IRM on 24 October, 1985: magnetic field measurements. From top to bottom: the GSM field components and the total field strength 
components $B_{y}$ and $B_{z}$ variations during this time are similar to the corresponding IMF components but amplified by a factor of about 9. The magnetosheath $B_{x}$ component, however, is not related to IMF $B_{x}$. During this time interval, when AMPTE/IRM is near the magnetopause, the magnetosheath $B_{x}$ component is a projection on the $\mathrm{x}$-axis of the compressed and draped magnetosheath magnetic field tangent to the magnetopause, and the latter is related only to the $B_{y}$ and $B_{z}$ components in the solar wind. (If the AMPTE/IRM orbit had coincided exactly with the $\mathrm{x}$-axis, the magnetosheath $B_{x}$ would decrease (to zero) as the magnetopause is approached (Spreiter et al., 1966).

Figure 4 displays magnetosheath flow quantities. From top to bottom the figure shows the angle between the flow vector and the normal to the model magnetopause $\left(\theta_{\mathrm{V}}, \mathrm{deg}\right)$; the $V_{z}, V_{y}$ and $V_{x}$ components of the flow vector ( $\mathrm{km} \mathrm{s}^{-1}$; GSM coordinates); and the bulk speed,
$V\left(\mathrm{~km} \mathrm{~s}^{-1}\right)$. The bulk speed decreases almost linearly along the orbit. At the magnetopause, the flow is directed along the magnetospheric boundary, as can best be seen in the top panel of Fig. 4, where a value of $\theta_{\mathrm{V}}=90^{\circ}$ (to the local magnetopause normal) is attained. As concluded by Phan et al. (1994), the linear decrease in $\theta_{v}$ across the magnetosheath is an indication of stationary conditions prevailing during this pass. As we saw already, this inference is supported by the ISEE 1 data.

A second feature, also noted by Phan et al. (1994) is a local enhancement of flow occurring from about $10 \mathrm{~min}$ prior to the magnetopause crossing, followed by a rapid decrease to the low values in the magnetosphere proper. The flows are accelerated mainly in the $y$ direction, i.e., roughly tangential to the magnetopause since the crossing is close to noon. In Fig. 5 we show a projection of the AMPTE/IRM orbit in the $(y z)$ plane with vectors drawn in representing the measured magnetic and
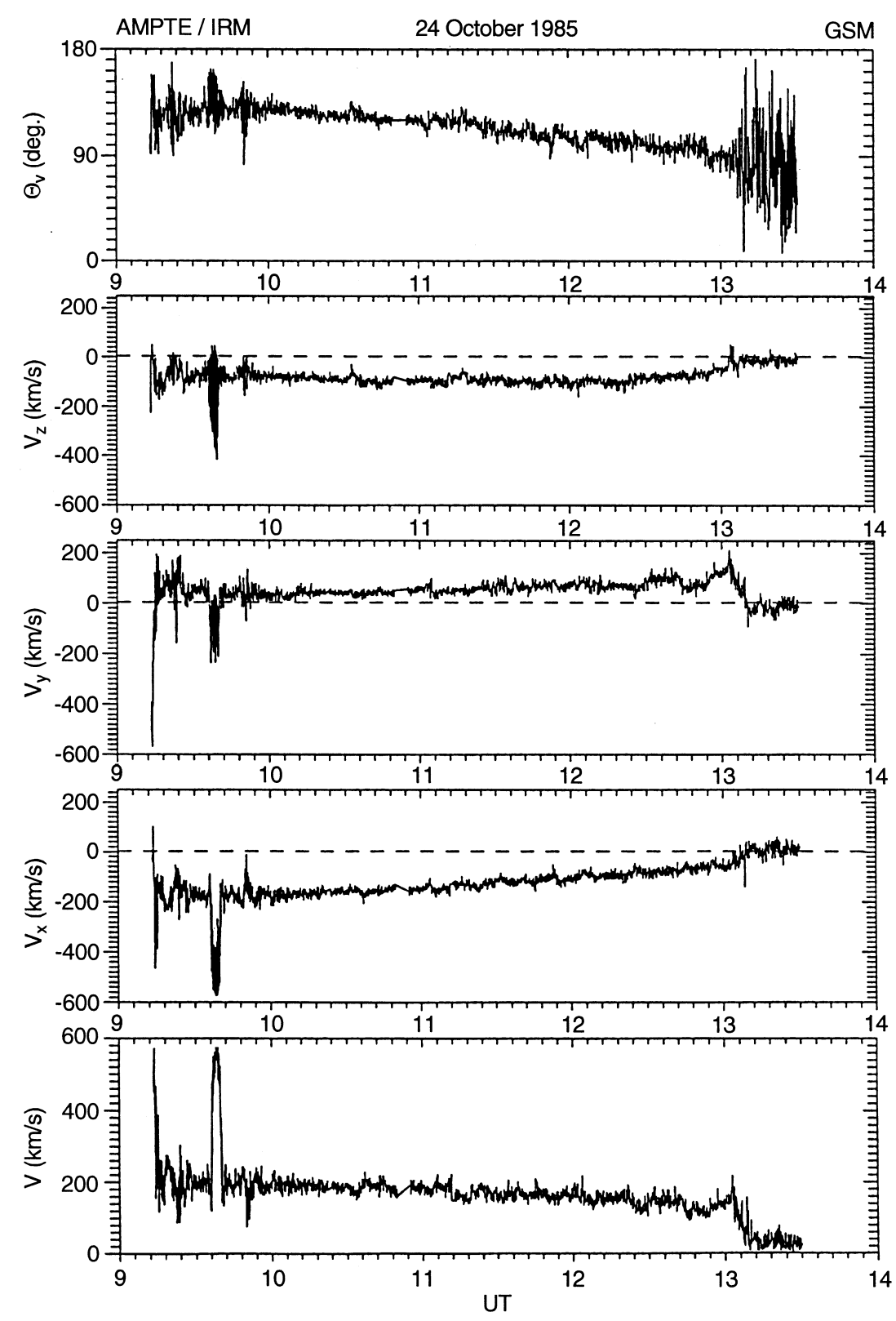

Fig. 4. AMPTE/IRM plasma flow observations on 24 October 1985: From top to bottom: the angle between the flow vector and the model magnetopause normal, the flow components $(G S M)$ and the bulk flow speed 


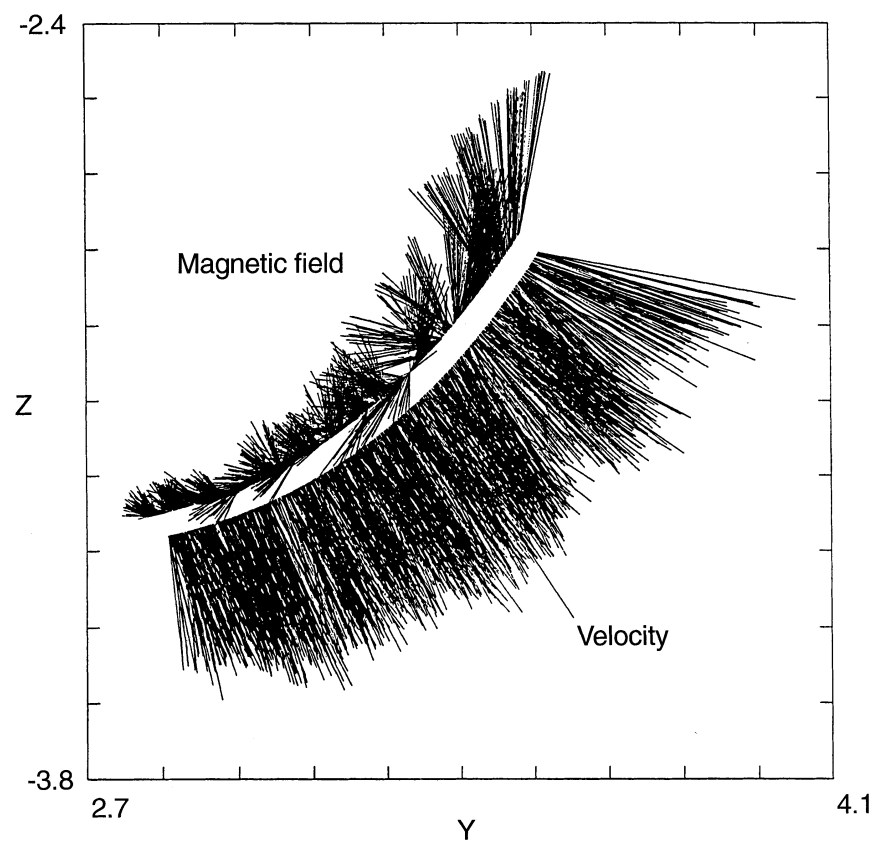

Fig. 5. A projection of the AMPTE/IRM trajectory in the $(Y Z)$ plane. The magnetopause crossing is at top right. The magnetosheath velocity and flow fields along the spacecraft trajectory are shown

velocity fields. The magnetopause crossing is at top right in the figure. The flow to the east (towards positive $y$ ) along the magnetopause and perpendicular to the local magnetosheath field direction is evident in this figure (see also Fig. 4 and Fig. 6), as is the progressive rotation of the magnetosheath plasma flow direction in the $(y z)$ plane as the spacecraft advances from the bow shock towards the magnetopause.

Further plasma and derived parameters are shown in Fig. 6 which displays, from top to bottom, the proton beta; the total pressure, $\Pi(\mathrm{nPa})$, defined already, the proton pressure, $P(\mathrm{nPa})$; the temperature, $T(\mathrm{~K})$; and the density, $N\left(\mathrm{~cm}^{-3}\right)$. The beta fluctuates strongly in the main body of the magnetosheath. After about 12:24 UT, however, the fluctuations decrease in amplitude and beta drops below unity (best seen in Phan et al., 1994, their Fig. 3). The total pressure increases steadily on average as the magnetopause is approached. After $\sim 12: 24 \mathrm{UT}$ this rise is due to the increase of magnetic pressure, discussed already, since the average gas pressure (panel 3 ) actually decreases. After $\sim 12: 24 \mathrm{UT}$, the temperature, too, shows a small decrease. A much more pronounced decrease, however, occurs in the density (last panel), which drops from average values of $16 \mathrm{~cm}^{-3}$ in the bulk of the magnetosheath to $\sim 2 \mathrm{~cm}^{-3}$ at the sunward side of the magnetopause. The behaviour of $N$ and $B$ near the magnetopause are defining characteristics of the PDL (see the Introduction). Following the work by Phan et al. (1994), we thus conclude that for $\sim 40$ min on this 4 -hour magnetosheath crossing, AMPTE/IRM encountered a PDL. Similar results are obtained if the plasma beta is used to define the PDL (Farrugia et al., 1995, 1997b), as explained later.

The flow enhancement and rotation mentioned earlier in connection with Fig. 4 and 5 are characteristic of
IRM results near crossings of the low-shear magnetopause. This is an MHD effect and would not be present if the magnetosheath flow were described by gas dynamics. A final point to note about the density profile is that, aside from high frequency fluctuations, present throughout the whole pass, there is no evidence on this pass of localized enhancements at, or around, the time when the density starts to decrease sharply near the magnetopause.

\section{Comparison of MHD model with observations}

We next compare the numerical MHD results with the data described in the previous section, starting with the parameters defining the PDL: $B$ and $N$. The solid lines in Fig. 7 represent the model variations. We calculate profiles along a straight-line pass in the ecliptic plane at 13:30 LT, which approximates the real IRM trajectory well. The input solar wind $B$ is that observed at $\sim 9: 12$ UT, when IRM is in the solar wind, while the input $N$ is an average of the values seen during the two excursions of IRM into the solar wind. The input solar wind Alfvén Mach number is 11.5 . We then use the duration of the pass and the assumption of quasi-stationarity to translate distances from the magnetopause to Universal Times. The comparison in Fig. 7 shows that theoretical variations of $N$ and $B$ match the measurements quite closely throughout the entire duration of the crossing, with shallow gradients in the outer and middle magnetosheath, becoming sharper in the inner magnetosheath. In particular, the field pile-up and density depression in the last $40 \mathrm{~min}$ of the pass are well reproduced. From 12:45 UT up to the time of the magnetopause crossing, the computed proton beta (not shown) lies below unity, again in good agreement with the observations (Fig. 6, top panel) and also in line with the depletion layer identification based on $N$ and $B$.

Figure 8 shows by solid lines the computed components of the flow vector in GSM coordinates. Upstream of the bow shock we have taken $V_{\infty}=(-550,0,0)$ $\mathrm{km} \mathrm{s}^{-1}$. Along our assumed orbit, the trends in the velocity component profiles are well reproduced, in particular the large and negative value of $V_{z}$. The enhancement in bulk speed at the magnetopause is clearly evident in the $V_{y}$ component, in agreement with the observations (see Fig. 4). However, we underestimate the absolute values of $V_{x}$ and $V_{z}$. We have taken the input solar wind from the IRM data prior to its bow shock crossing, in particular, in the present comparison of the magnetosheath flow components, we have taken $B_{y \infty}=0$ (see Fig. 3). However, the ISEE 1 data of Fig. 2 show a substantial negative $B_{y \infty}$ component. The plasma in the magnetosheath is accelerated orthogonal to the field, so this westward IMF component would lead, at the location of the AMPTE/IRM orbit, to an increase in the absolute value of $V_{z}$ and a slight decrease in $V_{y}$, if incorporated as input. (However, we cannot input ISEE 1 data because we need also the plasma to guarantee a reliable input to the model.) 
C. J. Farrugia et al.: MHD model of magnetosheath flow
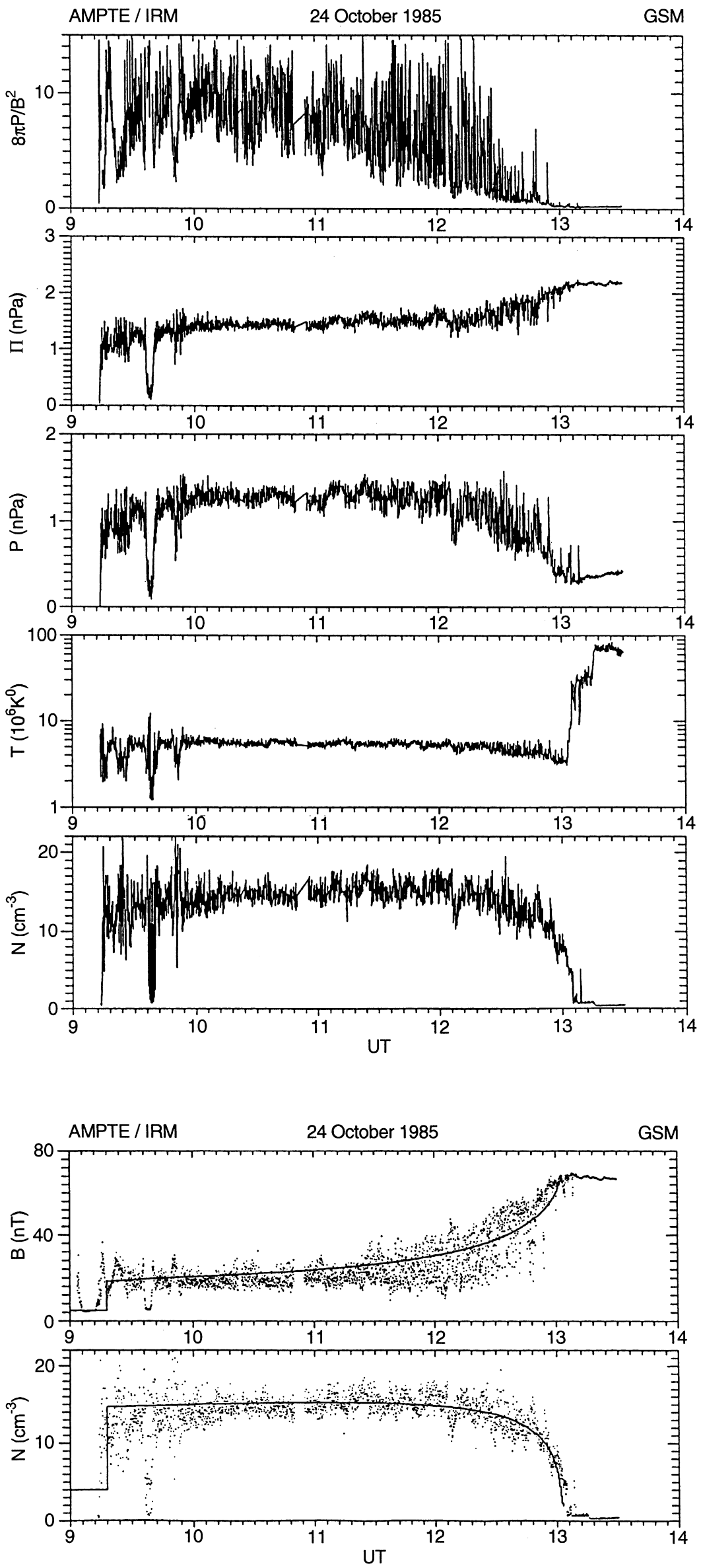

Fig. 6. For the same crossing as in Figs. 3 and 4, the panels show from top to bottom the proton beta, the sum of the proton and magnetic pressures, and the proton pressure, temperature and density
Fig. 7. The computed variations of the total field and the density across the magnetosheath (solid lines) are shown superimposed on the corresponding measurements (dots) 


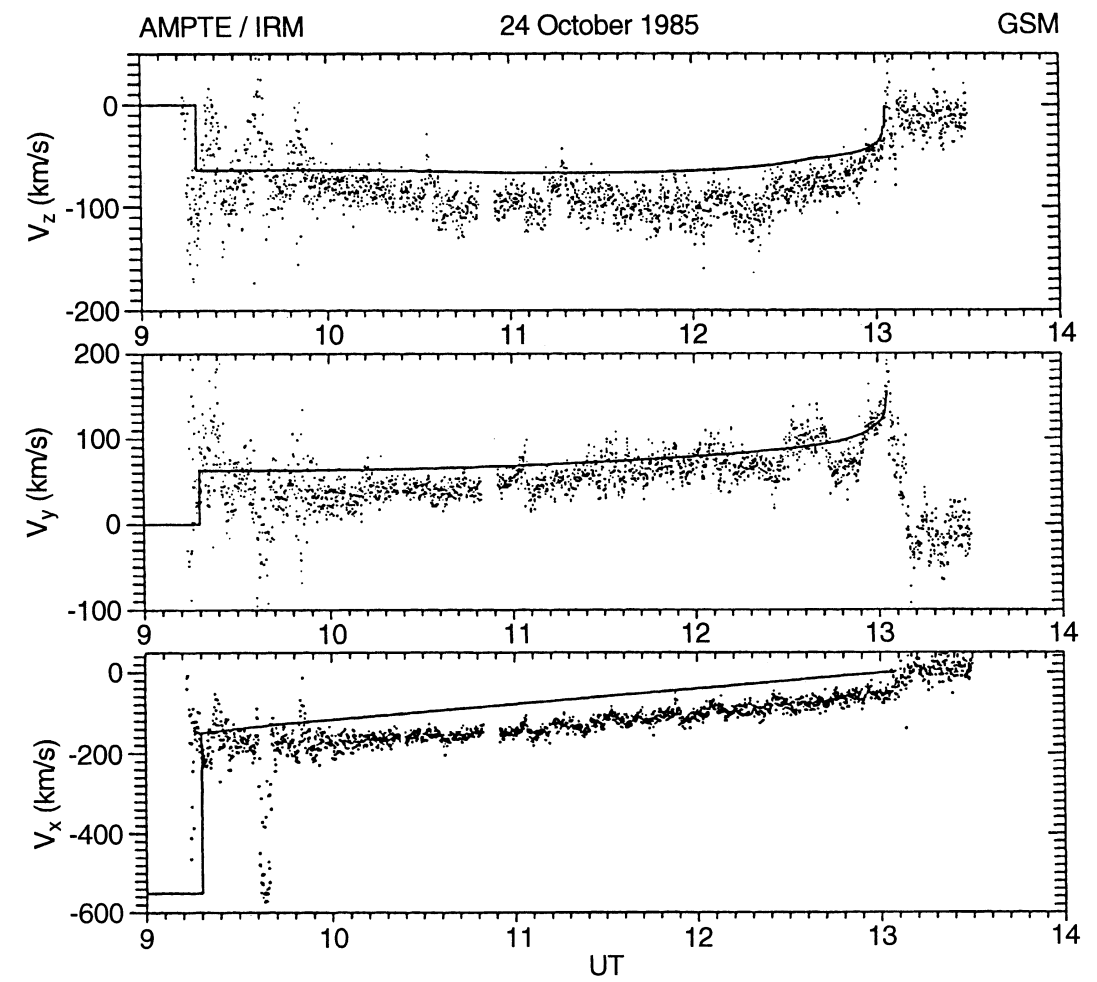

Fig. 8. The continuous lines show the computed variations in the flow components superimposed on the corresponding measurements
Figure 9 finally shows by a solid line in the top panel the angle between the computed velocity and magnetic fields across the magnetosheath, denoted by parameter $\alpha$. The data points give 10-point $(\sim 50 \mathrm{~s})$ averaged measurements of this quantity. The other panels display the temperature; and the total pressure, П. In the first panel, angle $\alpha$ is computed to be $102^{\circ}$ at the bow shock and $90^{\circ}$ at the magnetopause. The agreement between the trends in computed and average measured values of $\alpha$ is seen to be good throughout the magnetosheath, although the theoretical curve systematically overestimates the measurements by about $10^{\circ}$. While in the outer magnetosheath the average observed $\alpha$ is $\sim 90^{\circ}$ because the main components of $\mathbf{B}$ and $\mathbf{V}$ are $B_{z}$ and $V_{x}$,

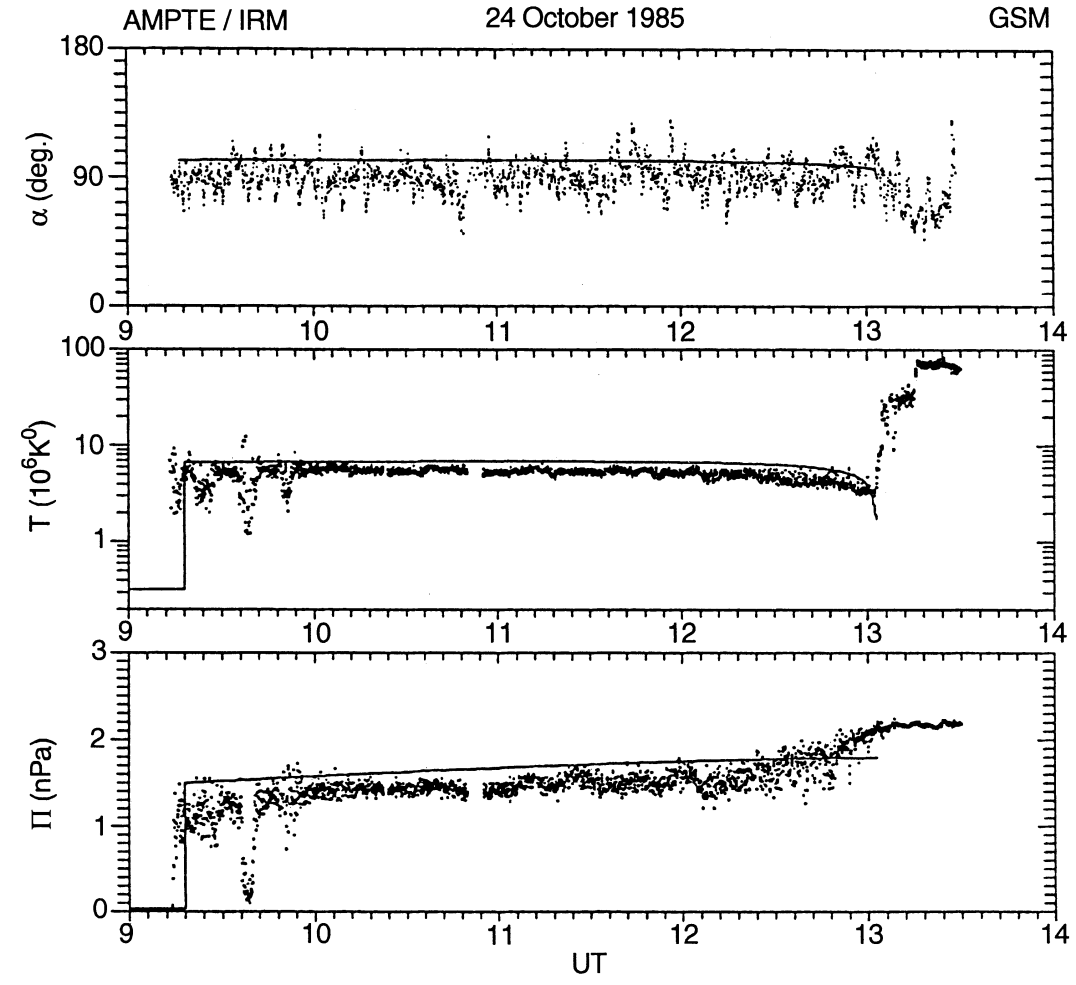

Fig. 9. The computed profiles of (from top to bottom) the angle between the flow and the field superimposed on the $\sim 50$ s-averaged measurements; the temperature; and the total pressure are shown superimposed on the measurements 
respectively, the orthogonality of flow and local magnetic field in the inner magnetosheath near the magnetopause reflects a PDL property. The modelled temperature, too, has the same trend as that measured, including a gentle decrease in the PDL. We think that the predicted stronger temperature drop right at the magnetopause with respect to observations may be removed once the isotropic pressure assumption is relaxed, but this is beyond the scope of the present paper. As noted in Sect. 2, a quadratic variation of the total pressure is assumed. This assumption refers to the variation of this quantity along a given normal to the magnetopause, which is the roughly model trajectory in this case. The last panel shows that the assumption is a fair one.

\section{Discussion and conclusions}

We compared a steady-state ideal MHD numerical model for flow in the terrestrial magnetosheath with observations during a steady magnetosheath crossing made by the AMPTE/IRM spacecraft on October 24, 1985. The comparison yielded a magnetosheath and PDL width in the Earth-Sun direction, as well as variations of field and flow parameters in both magnetosheath and PDL which compared satisfactorily with the data. In particular, the model predicts the presence of a PDL, which in this case was about $\sim 0.8 R_{E}$ thick in the Earth-Sun direction, in good agreement with the data (Phan et al., 1994). There is clear evidence of stagnation line flow features in the data, as predicted by the model.

In a recent study, Zhang et al. (1996) examined three traversals of the dayside magnetosheath in conjunction with simultaneous solar wind data. They distinguished three cases. In the first, the magnetosheath plasma and field variations were well correlated with solar wind changes when an appropriate time delay was included. Thus, they inferred that these magnetosheath observations were convected solar wind features. In the second case, the magnetosheath field was well correlated with the IMF but the plasma density was not, particularly in the inner magnetosheath. In the third case, neither the field nor the plasma in the inner magnetosheath were correlated with the corresponding interplanetary data. In particular, the magnetosheath data showed a localized density enhancement occurring near the sunward edge of the PDL at which the field and plasma pressures varied in antiphase. Zhang et al. (1996) suggested that these various magnetosheath changes are locally produced ("endogenous"). This interpretation corroborated earlier work by Song et al. (1990, 1992), who discovered this very interesting feature of density profiles in the inner magnetosheath. Song et al. (1992) interpreted these as a slow-mode wave transition. This feature has been the subject to a number of theoretical investigations, (e.g., Southwood and Kivelson, 1992; Omidi and Winske (1995) and others (see also Zhang et al., 1996 and references therein)).
This feature is not observed on the AMPTE/IRM pass we examined. The density and magnetic field profiles were smooth, with shallow gradients in the outer and middle magnetosheath, which become sharper near the magnetopause. Neither do we have this feature in our MHD model when (1) we work with isotropic pressure, (2) steady solar wind conditions are assumed, and (3) the magnetopause is a tangential discontinuity. This is not due to any numerical problem, such as finite grid size of the numerical scheme we used. As we saw, the agreement of the model with all aspects of the data is good.

In our opinion, this feature requires further study. There is as yet no generally accepted theoretical explanation of this interesting phenomenon. Neither do we think that the conditions under which this feature occurs have been unambiguously established. It might be that the density increase is somehow related to unsteady conditions. In this context it might not be coincidental that, studying magnetic field data from steady-state satellite passes across the Venus magnetosheath (where steady interplanetary conditions are easier to ascertain because of the small magnetosheath scale size relative to Earth), Luhmann (1995) found no evidence of a slow mode transition, but only a smooth variation corresponding to a simple depletion layer, as we did under comparable conditions.

Acknowledgements. This work is partially supported by NASA grant NAG5-2834, the Austrian "Fonds zur Förderung der wissenschaftlichen Forschung", grant P09431-TEC, and by grant 95-05-14192 from the Russian Foundation of Fundamental Research. CJF and NVE thank the Austrian Academy of Sciences for support during their visit to the Space Research Institute in Graz.

Topical Editor K.-H. Glaßmeier thanks D. H. Fairfield and D. Sibeck for their help in evaluating this paper.

\section{References}

Anderson, B. J., S. A. Fuselier, and D. Murr, Electromagnetic ion cyclotron waves observed in the plasma depletion layer, Geophys. Res. Lett., 18, 1955, 1991.

Biernat, H. K., G. A. Bachmaier, M. T. Kiendl, N. V. Erkaev, A. V. Mezentsev, C. J. Farrugia, V. S. Semenov, and R. P. Rijnbeek, Magnetosheath parameters and reconnection: a case study for the near-cusp region and the equatorial flank, Planet. Space Sci., 43, 1105, 1995.

Crooker, N. U., T. E. Eastman, and G. S. Stiles, Observations of plasma depletion in the magnetosheath at the magnetopause, J. Geophys. Res., 84, 869, 1979.

Erkaev, N. V., Results of the investigation of MHD flow around the magnetosphere, Geomagn. Aeron., 28, 455, 1988.

Farrugia, C. J., N. V. Erkaev, H. K. Biernat, and L. F. Burlaga, Anomalous magnetosheath properties during Earth passage of an interplanetary cloud, J. Geophys. Res., 100, 19 919, 1995.

Farrugia, C. J., N. V. Erkaev, H. K. Biernat, G. R. Lawrence, and R. C. Elphic, Plasma depletion layer model for low Alfvén Mach numbers: comparison with ISEE observations, J. Geophys. Res., 102, $11315,1997 \mathrm{a}$

Farrugia, C. J., N. V. Erkaev, H. K. Biernat, and L. F. Burlaga, Dependence of magnetosheath properties on solar wind Alfvén Mach number and magnetic shear across the magnetopause, in The solar wind-magnetosphere system 2, Eds. H. K. Biernat, 
H. P. Ladreiter, S. J. Bauer, C, J. Farrugia, p. 95, Austrian Academy of Sciences Press, Wien, 1997b.

Lees, L., Interaction between the solar wind plasma and the geomagnetic cavity, AIAA J., 2, 1576, 1964.

Luhmann, J. G., The inner magnetosheath of Venus: an analogue for Earth, J. Geophys. Res., 100, 12 035, 1995.

Midgley, J. E. and L. Davies, Calculation by a moment technique of the perturbation of the geomagnetic field by the solar wind, J. Geophys. Res., 68, 5111, 1963.

Omidi, N. and D. Winske, Structure of the magnetopause inferred from the Kinetic Riemann problem, J. Geophys. Res., 1995.

Paschmann, G., N. Sckopke, G. Haerendel, I. Papamastorakis, S. J. Bame, J. R. Asbridge, J. T. Gosling, E. W. Hones Jr., and E. R. Tech, ISEE plasma observations near the subsolar magnetopause, Space Sci. Rev., 22, 717, 1978.

Paschmann, G., W. Baumjohann, N. Sckopke, T.-D. Phan, and H. Lühr, Structure of the dayside magnetopause for low magnetic shear, J. Geophys. Res., 98, 13,409, 1993.

Petrinec, S. M., and C. T. Russell, Hydrodynamics and MHD equations across the bow shock and along the surfaces of planetary obstacles, Space Sci. Rev., 79, 757-791, 1997.

Phan, T.-D., and G. Paschmann, The magnetosheath region adjacent to the dayside magnetopause, in Physics of the Magnetopause, Ed. P. Song, B. U. O. Sonnerup, and M. F. Thomsen, $A G U$ Geophysical Monograph 90, p. 115, American Geophysical Union, Washington, D.C., 1995.
Phan, T.-D., G. Paschmann, W. Baumjohann, and N. Sckopke, The magnetosheath region adjacent to the dayside magnetopause: AMPTE/IRM observations, J. Geophys. Res., 99, 121, 1994.

Pudovkin, M. I., and V. S. Semenov, Stationary frozen-in coordinate system, Ann. Geophysi., 33, 429, 1977.

Song, P., C. T. Russell, J. T. Gosling, M. F. Thomsen, and R. C. Elphic, Observations of the density profile in the magnetosheath near the stagnation streamline, Geophys. Res. Lett., 17, 2035, 1990.

Song, P., C. T. Russell, and M. F. Thomsen, Slow mode transition in the frontside magnetosheath, J. Geophys. Res., 97, 8295, 1992.

Sonnerup, B. U. Ö., The reconnecting magnetopause, in Magnetospheric Physics, Ed. B. M. McCormac, p. 23, D. Reidel, Norwell, Mass., 1974.

Southwood, D. J., and M. G. Kivelson, On the form of the flow in the magnetosheath, J. Geophys. Res., 97, 2873, 1992.

Spreiter, J. R., A. L. Summers, and A. Y. Alskne, Hydrodynamic flow around the magnetosphere, Planet. Space Sci., 14, 223, 1966.

Zhang, X. X., P. Song, S. S. Stahara, J. R. Spreiter, C. T. Russell, and G. Le, Large-scale structures in the magnetosheath: exogenous or endogenous in origin?, Geophys. Res. Lett., 23, $105,1996$.

Zwan, B. J., and R. A. Wolf, Depletion of solar wind near a planetary boundary, J. Geophys. Res., 81, 1636, 1976. 